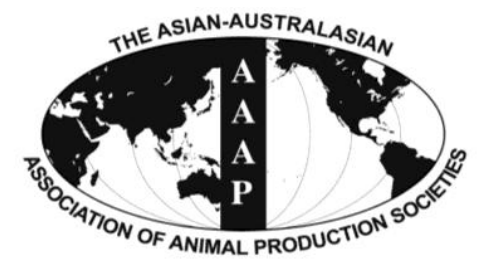

Asian-Aust. J. Anim. Sci.

Vol. 25, No. 7 : 950 - 955

July 2012

www.ajas.info

http://dx.doi.org/10.5713/ajas.2010.10355

\title{
In vitro-growth and Gene Expression of Porcine Preantral Follicles Retrieved by Different Protocols
}

\author{
J. I. Ahn ${ }^{1,2, a}$, S. T. Lee, ${ }^{3, a}$, J. H. Park ${ }^{1}$, J. Y. Kim ${ }^{4}$, J. H. Park ${ }^{1}$, J. K. Choi ${ }^{1}$, G. Lee ${ }^{5}$, E. S. Lee ${ }^{4}$ and J. M. Lim ${ }^{1,2, *}$ \\ ${ }^{1}$ WCU Biomodulation Program, Seoul National University, Seoul 151-742, Korea \\ ${ }^{2}$ Department of Agricultural Biotechnology, Seoul National University, Seoul 151-921, Korea
}

\begin{abstract}
This study was conducted to determine how the isolation method of the porcine preantral follicles influenced the following follicular growth in vitro. Mechanical and enzymatical isolations were used for retrieving the follicles from prepubertal porcine ovaries, and in vitro-growth of the follicles and the expression of folliculogenesis-related genes were subsequently monitored. The enzymatic retrieval with collagenase treatment returned more follicles than the mechanical retrieval, while the percentage of morphologically normal follicles was higher with mechanical retrieval than with enzymatic retrieval. After 4 days of culture, mechanically retrieved, preantral follicles yielded more follicles with normal morphology than enzymatically retrieved follicles, which resulted in improved follicular growth. The mRNA expression of FSHR, LHR Cx43, DNMT1 and FGFR2 genes was significantly higher after culture of the follicles retrieved mechanically. These results suggest that mechanical isolation is a better method of isolating porcine preantral follicles that will develop into competent oocytes in in vitro culture. (Key Words: Porcine, Preantral Follicle, Enzymatic Retrieval, Mechanical Retrieval, In vitro Culture, Follicular Growth)
\end{abstract}

\section{INTRODUCTION}

The importance of the pig in human regenerative medicine is based on its physiological similarity to humans (Hammer, 1998; Iverson and Talbot, 1998; Cozzi et al., 2009) which stimulates the development of porcine-specific reproductive biotechnology for human medicine. In fact, tremendous effort has been made to generate genetically engineering or cloned pigs for xenotransplantation and other medical purposes. In addition, a necessity to establish porcine stem cells is raised for supplying xenogenic tissues and organs. Securing of plenty number of developmentally competent oocytes is absolutely required to support porcine-oriented, novel biotechnology, but only the use of

\footnotetext{
* Corresponding Author: J. M. Lim. Tel: +82-2-880-4806, Fax: +82-2-874-2555, E-mail: limjm@snu.ac.kr

3 Department of Animal Biotechnology, Kangwon National University, Chunchon 200-701, Korea

${ }^{4}$ School of Veterinary Medicine and Institute of Veterinary Science, Kangwon National University, Chunchon 200-701, Korea.

${ }^{5}$ School of Dentistry, Seoul National University, Seoul 110-742, Korea.

${ }^{a}$ These two authors contribute equally to this work.

Submitted Oct. 4, 2010; Accepted Dec. 8, 2010; Revised Jul. 26, 2011
}

antral follicles in conventional assisted reproduction technology (ART) program cannot meet even the minimal requirement. Considering of such existing limitations, culture of porcine preantral follicles for deriving developmentally competent oocytes has been suggested, which enables to supply millions of immature follicles for reproductive purposes (Oxender et al., 1979; Telfer, 1996). Furthermore, establishment of the follicle culture system enables to further elucidate various mechanisms on folliculogenesis and oogenesis, which further accelerate the development of innovative biotechnology. Unfortunately, only limited success on deriving developmentally competent oocytes from the immature follicle culture has been reported to date (Wu et al., 2001; Wu et al., 2007a; Wu and Tian, 2007b).

Establishment of standard protocol of preantral follicle culture is now urged to establish and we have elaborated to develop an efficient protocol of follicle retrieval and subsequent culture for deriving developmentally competent embryos. In this study, we compared the effects of mechanical and enzymatic isolation of porcine follicles on in vitro-growth of preantral follicles. We subsequently investigated relative expression of folliculogenesis-related genes to select the optimal retrieval protocol for the follicle culture. 


\section{MATERIALS AND METHODS}

\section{Animal and tissue collection}

Ovaries were collected from prepubertal gilts at a slaughterhouse and transported to the laboratory in Dulbecco's phosphate-buffered saline (DPBS) supplemented with antibiotics at $37^{\circ} \mathrm{C}$.

\section{Preantral follicle retrieval and in vitro culture}

Ovaries were washed several times in fresh DPBS supplemented with antibiotics at $37^{\circ} \mathrm{C}$ prior to follicle retrieval. Regardless of retrieval method, the ovarian cortex was cut into slices 2 to $3 \mathrm{~mm}$ thick with microscissors. Ovarian follicles 4 to $6 \mathrm{~mm}$ in diameter were retrieved with a 26-gauge hypodermic needle from the dissected tissue (mechanical retrieval). Otherwise, the follicles were retrieved from the dissected tissues treated with 2,000 units (400 units/ml) of collagenase type I (Sigma-Aldrich, St. Louis, MO, USA) at $39^{\circ} \mathrm{C}$ with agitating for $30 \mathrm{~min}$ in a shaking incubator. The collected preantral follicles were washed in North Carolina State University (NCSU)-23 medium (Wu et al., 2001) supplemented with $3 \mathrm{mg} / \mathrm{ml}$ bovine serum albumin (BSA; Sigma-Aldrich) for $10 \mathrm{~min}$. The follicles were transferred singly onto 24-well dishes containing NCSU-23 medium supplemented with $10 \mathrm{~L} / \mathrm{ml}$ of insulin, transferrin, and selenium (ITS) solution (SigmaAldrich), $100 \mathrm{~g} / \mathrm{ml} \mathrm{L-ascorbic} \mathrm{acid} \mathrm{(Sigma-Aldrich),} \mathrm{10 \%}$ $(\mathrm{v} / \mathrm{v})$ porcine follicular fluid (pFF), and $200 \mathrm{mIU} / \mathrm{ml}$ follicle-stimulating hormone (FSH; Sigma-Aldrich) and cultured for $4 \mathrm{~d}$ in an humidified atmosphere containing $5 \%$ $\mathrm{CO}_{2}$ at $39^{\circ} \mathrm{C}$. Diameters of the cultured follicles were measured every $24 \mathrm{~h}$ using an inverted microscope.

The pFF used for follicle culture was extracted from antral follicles larger than 4 to $6 \mathrm{~mm}$ in diameter by centrifugation at $1,900 \times \mathrm{g}$ for $20 \mathrm{~min}$. The supernatant was subsequently filtered three times using filters with pore sizes of 1.2, 0.45 and $0.2 \mu \mathrm{m}$. After filtration, the $\mathrm{pFF}$ was used for follicle culture.

\section{In vitro maturation of oocyte-cumulus complex $(\mathrm{COCs})$}

To induce maturation of the intrafollicular oocytes, COCs were freed from the cultured follicles at the end of the culture (fourth day). Then, the isolated COCs were cultured in tissue culture medium (TCM)-199 (Gibco Invitrogen, Grand Island, NY, USA) supplemented with $0.23 \mathrm{mM}$ pyruvate (Sigma-Aldrich), $0.57 \mathrm{mM}$ cysteine (Sigma-Aldrich), $10 \%$ (v/v) pFF, $5 \mathrm{IU} / \mathrm{ml}$ equine chorionic gonadotropin (eCG; Intervet International BV, Boxmeer, The Netherlands), $5 \mathrm{IU} / \mathrm{ml}$ human chorionic gonadotropin (hCG; Intervet International BV), $20 \mathrm{mIU} / \mathrm{ml}$ ovine $\mathrm{FSH}$, and $10 \mathrm{ng} / \mathrm{ml}$ epidermal growth factor (EGF; SigmaAldrich) in the atmosphere containing $5 \% \mathrm{CO}_{2}$ at $39^{\circ} \mathrm{C}$ for $22 \mathrm{~h}$. Next, the COCs were transferred to eCG- and hCGfree medium and further cultured to allow the initiation and completion of meiotic maturation for $22 \mathrm{~h}$. The parameters of oocyte maturation were extrusion of a first polar body, expansion of cumulus cell layer, and formation of a metaphase II plate, which were confirmed by either immunostaining using Hoechst 33342 (Sigma-Aldrich) fluorescent dye and light microscopy.

\section{Quantitative analysis of mRNA expression}

At the beginning and end of follicle culture, the levels of FSH $(F S H R)$ and LH $(L H R)$ receptor, gap junction protein connexin-43 (Cx43), DNA methyltransferase (DNMT1), and fibroblast growth factor-2 receptor (FGFR2) mRNA expression in the follicles developing to the pseudoantral stage (Lee et al., 2008) were quantified by real-time PCR. Total RNA was extracted from the follicles according to the manufacturer's instructions and cDNA was synthesized using the Reverse Transcription System (Promega, Madison, WI). Primer3 software (Whitehead Institute, MIT Center for Genome Research, Cambridge, MA, USA) was used to design specific primers for $C \times 43$ and $F S H R$. The primers for $L H R, 18 \mathrm{~S}$ ribosomal RNA (18S), $D N M T 1$, and $F G F 2$ were described previously (Wu et al., 2006; Ozawa et al., 2008).

The sequences of PCR primers used are listed in Table 1. All results were normalized relative to $18 \mathrm{~S}$ RNA, and the relative RNA levels are presented as $2^{-\Delta \Delta \mathrm{Ct}}$, where $\mathrm{Ct}$ is the threshold cycle for target amplification,

$$
\begin{aligned}
& \Delta \mathrm{Ct}=\mathrm{Ct}_{\text {target gene }}-\mathrm{Ct}_{\text {internal reference }}(18 \mathrm{~S}) \text {, and } \\
& \Delta \Delta \mathrm{Ct}=\Delta \mathrm{Ct}_{\text {sample }}-\mathrm{Ct}_{\text {calibrator }}
\end{aligned}
$$

Table 1. Real time RT-PCR primer used

\begin{tabular}{lll}
\hline \multirow{2}{*}{ Gene } & \multicolumn{2}{c}{ Primer sequence } \\
\cline { 2 - 3 } FSHR & \multicolumn{1}{c}{ Sense $\left(5^{\prime}>3^{\prime}\right)$} & \multicolumn{1}{c}{ Antisense $\left(5^{\prime}>3^{\prime}\right)$} \\
$L H R$ & GATGTGATTTCTCCCTGAG & AAGGAACCGAGGGACTGTGA \\
$C x 43$ & GATAGAAGCTAATGCCTTTG & GGTATTTTAACCGAGGTAGA \\
$D N M T 1$ & CGCTTATTTCAATGGCTGCTC & AAAGGCTGTGCATGGGAGTTA \\
$F G F R 2$ & AGGTGAGGACATGCAGCTTT & AACTTGTTGTCCGTTGG \\
$18 S$ r $R$ RA & ATTCTGTGCCGGATGAAAGAC & GGTGTTGGAGTTCATGGAGG \\
\hline
\end{tabular}


Table 2. The number of morphologically normal follicles per ovary between mechanical method and enzymatic combined isolation method

\begin{tabular}{|c|c|c|c|c|c|}
\hline \multirow{2}{*}{$\begin{array}{l}\text { Methods of } \\
\text { isolation }\end{array}$} & \multicolumn{2}{|c|}{ Profiles of ovaries examined } & \multirow{2}{*}{$\begin{array}{l}\text { Total no. of follicles } \\
\text { retrieved }\end{array}$} & \multirow{2}{*}{$\%$ of normal follicles ${ }^{a}$} & \multirow{2}{*}{$\begin{array}{c}\text { No. of normal follicles } \\
\text { retrieved per ovary }\end{array}$} \\
\hline & Size $(\mathrm{cm})$ & Weight $(\mathrm{g})$ & & & \\
\hline Mechanical & $2.5 \pm 0.3$ & $2.9 \pm 0.7$ & $68 \pm 30$ & $95 \pm 4$ & $65 \pm 30$ \\
\hline Enzymatic & $2.5 \pm 0.3$ & $2.9 \pm 0.7$ & $128 \pm 58$ & $74 \pm 7$ & $96 \pm 44$ \\
\hline
\end{tabular}

Model effects of the treatment on total number of follicles retrieved, the percentage of normal follicles retrieved and number of normal follicles retrieved per ovary, which were indicated as $\mathrm{p}$ value, were 0.0207 , less than 0.0001 and 0.1228 , respectively.

${ }^{\mathrm{a}}$ The follicles existing intact basement membrane and integrated follicular structure were regarded as morphologically normal follicles.

\section{Experimental design and statistical analysis}

In vitro-growth of porcine preantral follicles retrieved with either mechanical or enzymatical method was monitored. Derivation of developmentally competent oocytes developing into the metaphase II stage was also evaluated after monitoring the follicle development. In the follicles retrieved by different method, FSHR, LHR, Cx43, $D N M T 1$ and FGFR2 expression of folliculogenesis-related genes were subsequently monitored. All experiments were replicated three times, and in the case of mRNA quantification, 30 follicles were equally divided into each replicate. Data are presented as mean $\pm \mathrm{SD}$, and statistical significance was determined by one-way ANOVA using SAS PROC-GLM (SAS Institute, Cary, NC, USA). In all statistical analyses, $\mathrm{p}<0.05$ was taken to indicate significance.

\section{RESULTS}

Comparison of mechanical and enzymatic isolation methods on follicle retrieval from porcine ovaries

Porcine ovaries of similar size $(2.5 \pm 0.3 \mathrm{~cm})$ and weight $(2.9 \pm 0.7 \mathrm{~g})$ were selected then ovarian follicles were retrieved with either mechanically or enzymatically. As shown in Table 2, a significant $(\mathrm{p}<0.05)$ difference between retrieval methods was detected in the total number of preantral follicles (68 vs. 128 follicles) and percentage of morphologically normal follicles (95\% vs. $74 \%$ ) retrieved. Enzymatic retrieval enabled better collection of preantral follicles, but the percentage of morphologically normal follicles was increased significantly with mechanical retrieval. The number of morphologically normal follicles retrieved per ovary was greater with enzymatic retrieval (96 vs. 65 follicles).

\section{In vitro growth of mechanically and enzymatically retrieved porcine preantral follicles}

Next, 100 morphologically normal follicles retrieved using each method were selected randomly and cultured under specific conditions. As shown in Table 3, the mean follicular size at retrieval did not differ significantly between two methods ( $233 \mu \mathrm{m}$ vs. $232 \mu \mathrm{m}$ ). However, the follicles retrieved with the mechanical method had significantly larger diameters than those retrieved with collagenase treatment on day $4(256 \mu \mathrm{m}$ vs. $243 \mu \mathrm{m}$; $\mathrm{p}<0.05)$. With the mechanical retrieval, the percentage of

Table 3. The diameters of preantral follicles cultured on day 4, percentage of follicles maintaining morphological integrity, number of follicles derived from diffuse oocytes, and number of intrafollicular oocytes undergoing germinal vesicle (GV) breakdown differed significantly between enzymatic and mechanical isolation

\begin{tabular}{lccc}
\hline \multirow{2}{*}{ Parameters } & Day of culture & \multicolumn{2}{c}{ Follicle isolation } \\
\cline { 3 - 4 } Number of preantral follicles cultured & - & With collagenase & Without collagenase \\
Diameter of follicles $(\mu \mathrm{m})$ & 0 & 100 & 100 \\
Diameter of follicles $(\mu \mathrm{m})$ & 1 & $232.72 \pm 8.5$ & $231.75 \pm 9.5$ \\
Diameter of follicles $(\mu \mathrm{m})$ & 2 & $237.62 \pm 6.4$ & $236.55 \pm 3.7$ \\
Diameter of follicles $(\mu \mathrm{m})$ & 3 & $240.04 \pm 7.3$ & $239.18 \pm 5.2$ \\
Diameter of follicles $(\mu \mathrm{m})$ & 4 & $242.36 \pm 8.3$ & $245.58 \pm 2.9$ \\
$\%$, follicles formed pseudoantrum & 2 & $243.05 \pm 9.2$ & $255.57 \pm 1.3 *$ \\
$\%$, follicles remained morphological integrity & 4 & $99 \pm 2.4$ & $100 \pm 0.0$ \\
$\%$, follicles derived diffused oocytes & 4 & $73 \pm 5.7$ & $95.2 \pm 4.2 * *$ \\
$\%$, oocytes underwent GV breakdown & 4 & $66.9 \pm 6.7$ & $84.0 \pm 6.4 * *$ \\
$\%$, oocytes matured & 4 & $1.5 \pm 3.7$ & $13.0 \pm 4.9 *$ \\
\hline
\end{tabular}

$* \mathrm{p}<0.05, * * \mathrm{p}<0.001$ 
the follicles maintaining morphological integrity was higher than with the enzymatic retrieval (95\% vs. $73 \%$ ). The percentages of the follicles yielding diffused oocytes $(84 \%$ vs. $67 \%$ ), which is ready for initiating meiotic maturation and expanding cumulus cell matrix, and the number of oocytes undergoing germinal vesicle breakdown (13\% vs. $2 \%$ ) were also higher with mechanical retrieval. Only oocytes derived from the follicles retrieved mechanically could be matured by induction, although the efficiency was as low as $1 \%$. The disruption of the basement membrane was detected in considerable number of the follicles retrieved enzymatically, while no detection was made in the enzymatically retrieved follicles (Figure 3).

Differential mRNA expression pattern of follicular developmental genes in differentially isolated porcine follicles

Relative mRNA expression of follicular hormone receptor, gap junction, and follicular development genes were investigated. At the beginning of culture, no significant differences between two retrieval methods were detected except for Dnmt3 (Figure 1). On day 4 of culture, however, expression of all genes evaluated was significantly elevated in mechanically retrieved follicles compared with enzymatically retrieved follicles (Figure 2).

\section{DISCUSSION}

The results of this study clearly demonstrate that the follicle-retrieval procedure has a great impact on postretrieval survival and further growth follicles in vitro, which influences the derivation of developmentally competent oocytes following in vitro culture. The retrieval of preantral follicles without enzymatic (collagenase) treatment resulted in better follicular growth and oocyte maturation than did the enzymatic retrieval of the follicles. One of the major factors responsible for this effect may be related to maintaining the structural integrity of follicles during isolation and afterward, which results in the maintenance of normal expression by folliculogenesis-related genes directly. Numerous reports have addressed the importance of the cellular network and coordination between follicular cells and intrafollicular oocytes for acquiring developmental competence (Eppig et al., 1996; Gougeon, 1996; Orisaka et al., 2009; Su et al., 2009). In this study, damage to the basement membrane of the follicles was frequently seen a

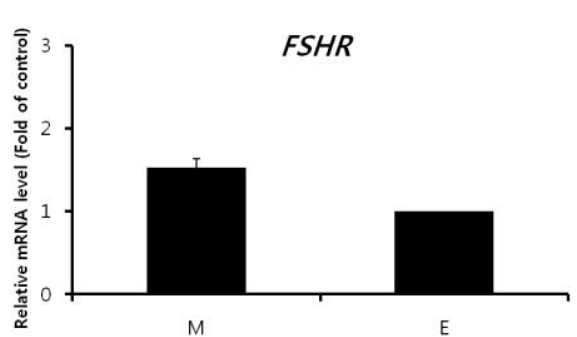

C

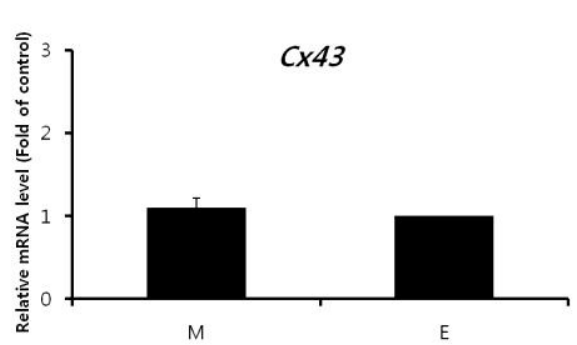

e

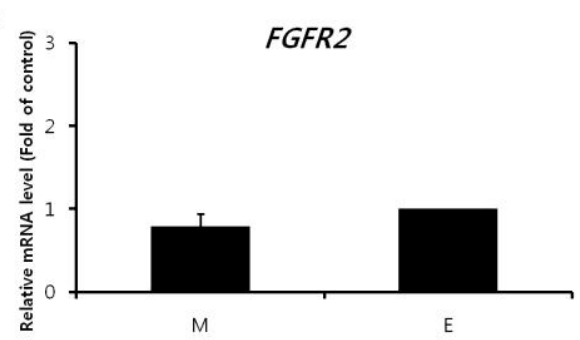

b

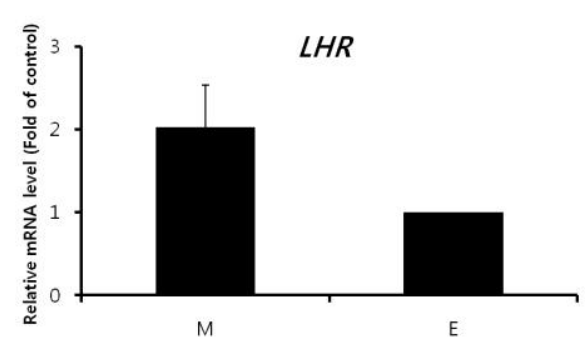

d

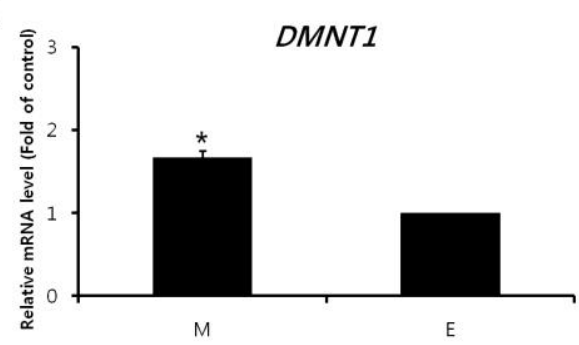

Figure 1. Gene expression of porcine preantral follicles isolated using a mechanical method alone or mechanical retrieval combined with collagenase treatment. Expression of the (a) FSH receptor (FSHR), (b) LH receptor (LHR), (c) gap junction protein (Cx43), and folliculogenesis-related (d) DNMT1 and (e) FGFR2 genes in the follicles retrieved with mechanical (M) or enzymatic (E) treatment. Data are given as the mean $\pm \mathrm{SE}$ of three replicates. $* \mathrm{p}<0.05$. 
a
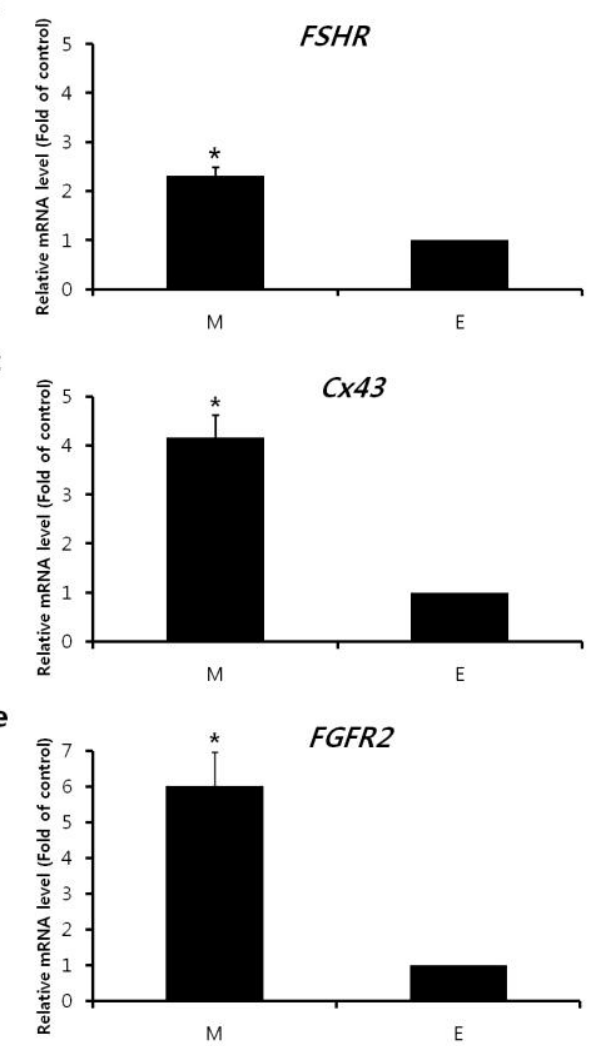

b

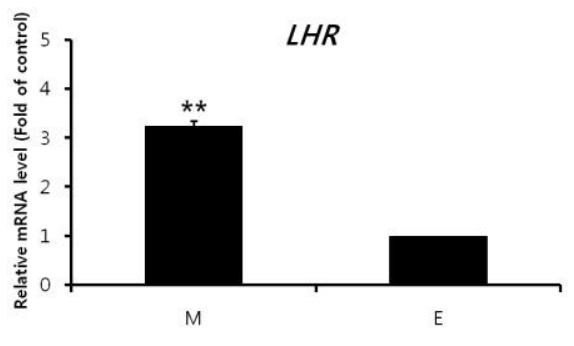

d

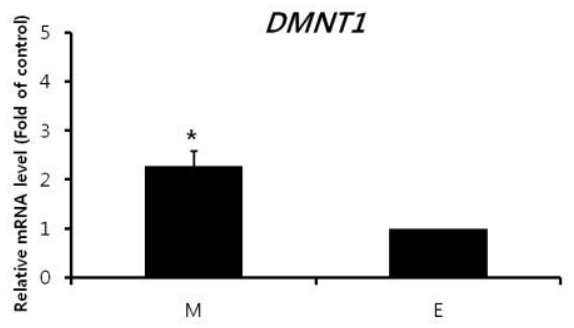

Figure 2. Gene expression of porcine preantral follicles cultured in vitro for 4 days. Follicles isolated using a mechanical method alone or mechanical retrieval combined with collagenase treatment. Expression of the (a) FSH receptor (FSHR), (b) LH receptor (LHR), (c) gap junction protein (Cx43), and folliculogenesis-related (d) DNMT1 and (e) FGFR2 genes in the follicles retrieved with mechanical (M) or enzymatic (E) treatment. Data are given as the mean \pm SE of three replicates. ${ }^{*} \mathrm{p}<0.05$, ${ }^{* *} \mathrm{p}<0.001$.
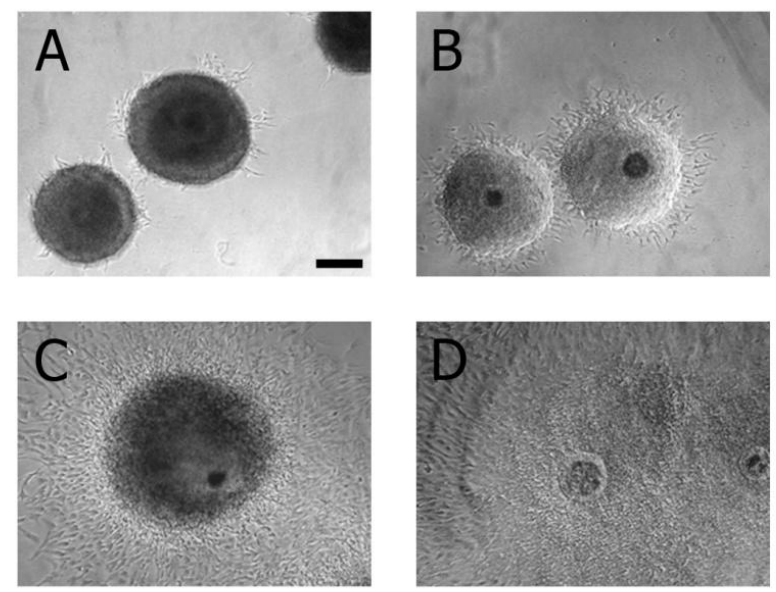

Figure 3. Breakdown of the basement membrane of porcine preantral follicles during in vitro culture. Preantral follicles were enzymatically isolated from the ovaries and subsequently cultured in vitro. Morphology of the follicles were examined every day during the culture. (A) day 1, (B) day 2, (C) day 3 and (D) day 4. After day 2 of the culture, basement membrane was detached from the surface of follicle (B) and then the follicle was ruptured (C). Bar $=100 \mu \mathrm{m}$. after the enzymatic treatment, which supports the importance of the structural integrity of follicles for follicle growth.

Structural damage induced by enzymatic treatment negatively influences the activation of mRNA expression in cultured follicles, which decreases the derivation of developmentally competent oocytes. However, only morphologically normal follicles developing to the same stage were provided for quantitative RT-PCR. With the randomized allocation of collected follicles to each treatment, no difference in gene expression except for Dnmt3 was detected at the beginning of culture (Figure 1). Therefore, accumulative damages in addition to reducing structural integrity of the follicles were triggered by the enzymatic treatment at the time of retrieval. These may inhibit the activation of genes related to gonadotropin receptors, extracellular matrix protein, and follicle growth in preantral follicles cultured in vitro (Demeestere et al., 2002; Carrell et al., 2005). Enzyme treatment at the time of follicle retrieval probably further induces various changes in the mechanism regulating follicle growth and oocyte maturation.

Although mechanical retrieval further supports in vitro- 
folliculogenesis, it significantly reduced the number of retrieved follicles. In this study, only the expression of folliculogenesis-related genes were monitored, while further screening on the expression of the genes regulating cell stress and death may provide a cue to develop an alternative strategy for optimizing the retrieval of developmentally competent, preantral follicles from porcine ovaries. On the other hand, our improved retrieval method did not result in a significant increase in the production of mature oocytes. Considerable discrepancy in the outcome of porcine preantral follicle culture is often observed, despite using similar protocols (Wu et al., 2001; Mao et al., 2002; Mao et al., 2004; Wu et al., 2007a; Wu and Tian, 2007b). We postulate that miscellaneous differences among the culture systems result in the unpredictable outcomes. A simple comparison of the percentile values obtained with each culture condition is not appropriate for selecting the optimized protocol, and it may be necessary to combine protocols to establish an optimized system. The culture protocol should further be optimized and standardized. At least, the information from our study can contribute to optimizing preantral culture protocol by efficient supporting in vitro-development of preantral follicles as well as by sufficient retrieving large number of developmentally competent oocytes.

\section{ACKNOWLEDGEMENT}

This work was supported by a grant (052520110037) from Animal, Plant \& Fisheries Quarantine and Inspection Agency (QIA), Ministry of Food, Agriculture, Forestry, and Fisheries, and by a grant (\#20080401034072) from the BioGreen 21 Program, Rural Development Administration, Republic of Korea. The authors also acknowledge a world class university (WCU) program (R31-10056) on Biomodulation, which is supported by Ministry of Education, Science and Technology, Republic of Korea.

\section{REFERENCES}

Carrell, D. T., L. Liu, I. Huang and C. M. Peterson. 2005. Comparison of maturation, meiotic competence, and chromosome aneuploidy of oocytes derived from protocols for in vitro culture of mouse secondary follicles. J. Assist. Reprod. Genet. 9-10:347-534.

Cozzi, E., E. Basio, M. Seveso, D. Rubello and E. Ancona. 2009. Xenotransplantation as a model of integrated, multidisciplinary research. Organogenesis 5:288-296.

Demeestere, I., A. Delbaere, C. Gervy, M. Van Den Bergh, F. Devreker and Y. Englert. 2002 Effect of preantral follicle isolation technique on in-vitro follicular growth, oocyte maturation and embryo development in mice. Hum. Reprod. 17:2152-2159.

Eppig, J. J., M. O'Brien and K. Wigglesworth. 1996. Mammalian oocyte growth and development in vitro. Mol. Reprod. Dev.
44:260-273.

Gougeon, A. 1996. Regulation of ovarian follicular development in primates: facts and hypotheses. Endocr. Rev. 17:121-155.

Hammer, C. 1998. Physiological obstacles after xenotransplantation. Ann. NY Acad. Sci. 862:19-27.

Iverson, W. O. and T. Talbot. 1998. Definition of production specification for xenotransplantation. Ann. NY Acad. Sci. 862: 121-124.

Lee, S. T., M. H. Choi, E. J. Lee, S. P. Gong, M. Jang, S. H. Park, H. Jee, D. Y. Kim, J. Y. Han and J. M. Lim. 2008. Establishment of autologous embryonic stem cells derived from preantral follicle culture and oocyte parthenogenesis. Fertil. Steril. 90:1910-1920.

Mao, J., G. Wu, M. F. Smith, T. C. McCauley, T. C. Cantley, R. S. Prather, B. A. Didion and B. N. Day. 2002. Effects of culture medium, serum type, and various concentrations of folliclestimulating hormone on porcine preantral follicular development and antrum formation in vitro. Biol. Reprod. 67:1197-1203.

Mao, J., M. F. Smith, E. B. Rucker, G. M. Wu, T. C. McCauley, T. C. Cantley, R. S. Prather and B. N. Day. 2004. Effect of epidermal growth factor and insulin-like growth factor I on porcine preantral follicular growth, antrum formation, and stimulation of granulosa cell proliferation and suppression of apoptosis in vitro. J. Anim. Sci. 82:1967-1975.

Orisaka, M., K. Tajima, B. K. Tsang and F. Kotsuji. 2009. Oocytegranulosa-theca cell interaction during preantral follicular development. J. Ovarian Res. 9:2-9.

Oxender, W. D., B. Colenbrander, D. F. M. Van de Wiel and C. J. G. Wensing. 1979. Ovarian development in fetal and prepubertal pigs. Biol. Reprod. 21:715-721.

Ozawa, M., T. Nagai, T. Somfai, M. Nakai, N. Maedomari, M. Fahrudin, N. W. Karja, H. Kaneko, J. Noguchi, K. Ohnuma, N. Yoshimi, H. Miyazaki and K. Kikuchi. 2008. Comparison between effects of 3-isobutyl-1-methylxanthine and FSH on gap junctional communication, LH-receptor expression, and meiotic maturation of cumulus-oocyte complexes in pigs. Mol. Reprod. Dev. 75:857-866.

Su, Y. Q., K. Suqiura and J. J. Eppig. 2009. Mouse oocyte control of granulose cell development and function: paracrine regulation of cumulus cell metabolism. Semin. Reprod. Med. 27:32-42.

Telfer, E. E. 1996. The development of methods for isolation and culture of preantral follicles from bovine and porcine ovaries. Theriogenology 45:101-110.

Wu, D., Q. C. Cheung, L. Wen and J. Li. 2006. A growthmaturation system that enhances the meiotic and developmental competence of porcine oocytes isolated from small follicles. Biol. Reprod. 75:547-554.

$\mathrm{Wu}$, J., and Q. Tian. 2007b. Role of follicle stimulating hormone and epidermal growth factor in the development of porcine preantral follicle in vitro. Zygote 15:233-240.

Wu, J., B. R. Emery and D. T. Carrell. 2001. In vitro growth, maturation, fertilization, and embryonic development of oocytes from porcine preantral follicles. Biol. Reprod. 64:375381.

Wu, J., B. Xu and W. Wang. 2007a. Effects of luteinizing hormone and follicle stimulating hormone on the developmental competence of porcine preantral follicle oocytes grown in vitro. J. Assist. Reprod. Genet. 24:419-24. 